Algebraic $\& \mathcal{G}$ Geometric Topology

Volume 1 (2001) 349-368

Published: 2 June 2001

ATG

\title{
A characterization of shortest geodesics on surfaces
}

\author{
Max Neumann-Coto
}

\begin{abstract}
Any finite configuration of curves with minimal intersections on a surface is a configuration of shortest geodesics for some Riemannian metric on the surface. The metric can be chosen to make the lengths of these geodesics equal to the number of intersections along them.
\end{abstract}

AMS Classification 53C22; 53C42,57R42

Keywords Surfaces, curves, geodesics, minimal intersections, metrics

If $S$ is a closed surface, with some Riemannian metric, then each essential curve immersed in $S$ is freely homotopic to a smooth geodesic in $S$ which is shortest among all the curves in that homotopy class. So while closed geodesics represent the critical points of the length in each free homotopy class, these shortest geodesics represent the absolute minima in each class. Shortest geodesics have topological properties which are not shared by all geodesics: Freedman, Hass, Rubinstein and Scott showed in [1] and [2] that shortest geodesics intersect minimally, i.e., they have the minimum number of intersections and self-intersections allowed by their free homotopy classes, unless they factor through coverings of other shortest geodesics. The curve in figure 1a, for example, represents a geodesic for some metric on $S$, but it can't represent a shortest geodesic. On the other hand, when a homotopy class allows different configurations with minimal intersections, as in figures $1 \mathrm{~b}, 1 \mathrm{c}$ and $1 \mathrm{~d}$, it seems natural to ask which ones correspond to shortest geodesics for some Riemannian metric on $S$. This question was first considered by Shepard [5].

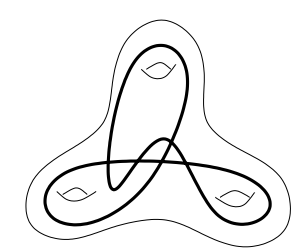

a

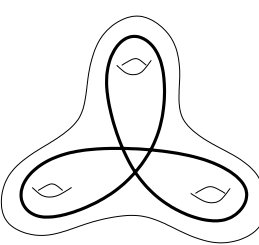

b

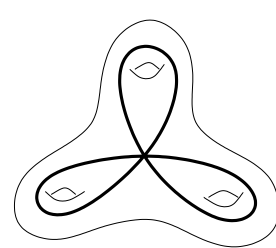

$\mathrm{C}$

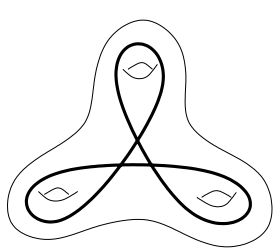

d

Figure 1

(c) Geometry $\&$ Topology Publications 
In this paper we prove that the shortest geodesics in a surface are characterized by the minimal intersection property, showing that any finite configuration of curves with minimal intersections in $S$ is a configuration of shortest geodesics for some Riemannian metric $g$ on $S$, and also that $g$ can be chosen to make the lengths of these geodesics equal to the number of intersections along them. The proof starts by 'blowing up' the metric outside a regular neighborhood of the curves (an idea introduced by Bonahon in the context of least area surfaces in 3-manifolds) to transform the problem into a combinatorial one.

The main result implies that all minimal configurations in $S$ can be extended to contain curves in any other homotopy classes, and gives conditions for the existence of 'absolute' inequalities relating the minimal lengths of curves in different homotopy classes (inequalities that hold for all Riemannian metrics on $S$ ). In the second part the idea of transmitting cut and paste instructions along a homotopy is combined with a result of Hass and Scott [3] to give a new proof of the minimal intersection property of [1] and to find some absolute inequalities involving minimal configurations.

\section{Minimal configurations.}

Two collections of immersed curves in $S$ have the same configuration if there is an ambient isotopy that moves the image of one to the other. The curves in a configuration intersect minimally or have minimal intersections if they minimize the number of intersections and self-intersections among all transverse and selftransverse curves in their free homotopy classes. Following [1] and [2], the intersections and self-intersections are counted 'in the source', by counting how many curves one crosses when following a curve all the way around (so multiple intersections are counted with multiplicity and all the curves in figure 1 have 6 self-intersections).

As with all geodesics in a surface, shortest geodesics are transverse and selftransverse, unless they factor through coverings of other geodesics. Shortest geodesics may not be unique, and they may not be in general position as they may have points of multiple intersections.

The results of [1] and [2] can be summarized as follows:

(a) Shortest geodesics intersect minimally, unless they are coverings of other shortest geodesics.

(b) If $\alpha$ is an orientation-preserving curve in $S$, the shortest geodesics representing powers of $\alpha$ always cover a shortest geodesic representing $\alpha$, but if $\alpha$ 
is orientation-reversing and there are 2 different shortest geodesics representing $\alpha$, then the shortest geodesics representing $\alpha^{2}$ and the odd powers of $\alpha$ do not cover other shortest geodesics.

According to these results, the image of a collection of shortest geodesics in $S$ is a configuration of essential curves that intersect transversely and minimally and do not represent proper powers of any orientation-preserving class. A finite configuration of essential curves in $S$ with these properties will be called a minimal configuration in $S$.

Theorem 1.1 Any minimal configuration of curves in a closed surface $S$ is a configuration of shortest geodesics for some Riemannian metric g on $S$. g can be chosen so that the length of each curve in the configuration is equal to the total number of intersections along it.

Remark The curves need not be in general position, some may be homotopic or represent proper powers of an orientation-reversing class. The curves with no intersections will have length 1.

Lemma 1.2 If $c_{1}, c_{2}, \ldots, c_{n}$ is a collection of curves immersed transversely in $S$, and $N$ is a regular neighborhood of $\cup c_{i}$, then there is a Riemannian metric $g$ on $S$ such that:

(a) Each $c_{i}$ is a geodesic in $S$ and a shortest geodesic in $N$.

(b) All essential curves in $S$ that don't lie entirely in $N$ are longer than every $c_{i}$.

(c) The lengths of the arcs of the configuration (the components of $\bigcup c_{i}-$ intersections) can be chosen to be any positive numbers.

Proof The idea is to make the surface look like a landscape with the curves lying in the bottom of deep and narrow canyons and surrounded by large mountains. Start with any Riemannian metric $g_{S}$ on $S$ and regular neighborhoods $N^{-} \subset N \subset N^{+}$of $\bigcup c_{i}$. Since there is a positive lower bound for the lengths of all essential curves in $S-N^{-}$and all arcs running from $S-N$ to $N^{-}$, then by multiplying $g_{S}$ by a constant $k$ we can make that lower bound larger than the desired lengths of the curves (this makes the canyons deep and the mountains large). Now we want to modify the metric inside $N^{+} . \bigcup c_{i}$ is a union of arcs that meet at the multiple points, so $N^{+}$is the union of (topological) rectangles and polygons (around the multiple points) as in figure 2a. Put a flat metric $g_{N}$ on $N^{+}$to make these rectangles and polygons Euclidean, so each $c_{i}$ is a geodesic and no homotopy of $c_{i}$ within $N^{+}$reduces its length. Since the lengths 
of the rectangles can be chosen independently of each other, we can choose the length of each arc of the configuration to be any positive number, and the diameters of the polygons can be taken to be smaller than any prescribed number $d$ (this makes the canyons long and narrow).

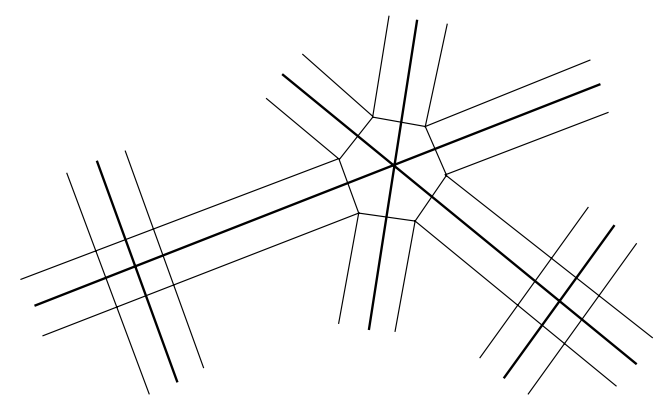

a

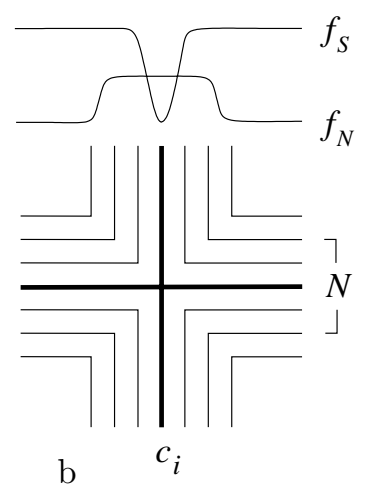

$\mathrm{b}$

Figure 2

Let $g=f_{S} g_{S}+f_{N} g_{N}$ (in terms of the first fundamental forms), where $f_{S}$ and $f_{N}$ are smooth scalar functions such that:

$f_{N}=1$ on $N, f_{N}>0$ on $N^{+}-N$ and $f_{N}=0$ on $S-N^{+}$.

$f_{S}=0$ on $\bigcup c_{i}, f_{S}>0$ on $N^{-}-\bigcup c_{i}$ and $f_{S}=k$ on $S-N^{-}$.

See figure 2b. As $g \geq k g_{S}$ on $S-N^{-}$, with the metric $g$ any essential curve in $S-N^{-}$and any arc that crosses from $N^{-}$to $S-N$ is longer than $c_{i}$, so any essential curve which doesn't lie in $N$ is longer than $c_{i}$. As $c_{i}$ was a shortest geodesics in $N$ with the metric $g_{N}$, and $g=g_{N}$ on $\bigcup c_{i}$ but $g>g_{N}$ on $N-\bigcup c_{i}$, the metric $g$ makes every $c_{i}$ a geodesic in $S$ and shortest geodesic in $N$.

Each essential curve $c$ immersed in $N$ is freely homotopic in $N$ to a polygonal curve $p$ made of arcs of the configuration (maybe repeated) and we may assume that $p$ is reduced in the sense that no arc is followed immediately by the same arc in the opposite direction.

Lemma 1.3 The metric $g$ can be defined so that if a (reduced) polygonal curve $p$ is longer than $c_{i}$, then all the curves homotopic to $p$ in $N$ are also longer than $c_{i}$.

Proof This is accomplished by choosing $d$ small (narrow canyons). If $p$ has corners (i.e., if $p$ is not one of the $c_{i}$ 's) then its length can be reduced by 
rounding the corners, but no homotopy within $N$ can reduce its length by more than $d$ multiplied by the number of corners of $p$ (this is clear for the metric $g_{N}$, and $g=g_{N}$ along $p$ and $g \geq g_{N}$ elsewhere in $N$ ), and the number of corners in $p$ is bounded above by a linear function of its length. So if $c$ is homotopic to $p$ in $N$ then $\frac{\text { length }(c)}{\text { length }(p)}>1-l d$, where $l$ is the coefficient of the linear function, so by taking $d$ small enough we can make this ratio as close to 1 as we want. But the set of lengths of the polygonal curves in $N$ is discrete (because it is contained in the set of positive linear combinations of the lengths of the arcs), so $\frac{\operatorname{length}\left(c_{i}\right)}{\text { length }(p)}<m<1$ for all $c_{i}$ 's and all longer $p$ 's. So by taking $d$ small we can make $\frac{\text { length }(c)}{\text { length }(p)}>\frac{\operatorname{length}\left(c_{i}\right)}{\operatorname{length}(p)}$ for every $p$ longer than $c_{i}$.

The previous lemmas have no minimal intersection hypothesis: the metric $g$ makes each $c_{i}$ a geodesic in $S$, but not necessarily a shortest geodesic, because nonhomotopic curves in $N$ may be homotopic in $S$. The $c_{i}$ 's are shortest geodesics for some metric $g$ on $S$ if and only if the lengths of the arcs of the configuration can be chosen so that all homotopic $c_{i}$ 's have the same length and all polygonal curves homotopic to $c_{i}$ are longer than $c_{i}$.

Now let $c_{1}, c_{2}, \ldots, c_{n}$ be a collection of curves with minimal intersection and selfintersection in $S$. In order to choose the lengths of the arcs of the configuration, take a collection of measuring curves $\mu_{1}, \mu_{2}, \ldots, \mu_{m}$ in general position with respect to the $c_{i}$ 's, assign to each $\mu_{j}$ a positive width $w_{j}$, and define the length of each arc of the configuration as the sum of the widths of the curves $\mu_{j}$ that meet the arc. As the arcs of the configuration must have positive length, we need a collection $\left\{\mu_{j}\right\}$ whose union meets all the arcs.

Let's say that a measuring collection for $\left\{c_{i}\right\}$ is good if it intersects each $c_{i}$ minimally but does not intersect any polygonal curve homotopic to some $c_{i}$ minimally.

Lemma 1.4 If $\left\{\mu_{j}\right\}$ is a good measuring collection, then for any choice of widths the assigned lengths make the $c_{i}$ 's shortest geodesics for a Riemannian metric in $S$.

Proof As the $\mu_{j}$ 's intersect $c_{i}$ minimally, if a polygonal curve $p$ is homotopic to $c_{i}$, then each $\mu_{j}$ must intersect $p$ at least as many times as it intersects $c_{i}$, so $p$ is at least as long as $c_{i}$, and it is longer than $c_{i}$ if and only if the total number of intersections of the $\mu_{j}$ 's with $p$ is larger, i.e., if some $\mu_{j}$ does not intersect $p$ minimally. This is clearly independent of the choice of widths. Now apply lemmas 1.2 and 1.3 . 
Remark Notice that if a good measuring collection is extended in any way (by adding curves that intersect the $c_{i}$ 's minimally) then the resulting measuring collection is good.

\section{Construction of good measuring collections}

Let $\left\{c_{i}\right\}$ be a configuration of essential curves in a surface $S$. If $\chi(S) \leq 0$, the universal covering of $S$ is a plane $\widetilde{S}$, and the cyclic coverings $S^{\alpha}$ of $S$ corresponding to the subgroups generated by elements $\alpha$ of $\pi_{1}(S)$ are annuli or Moebius bands (depending on whether $\alpha$ is orientation preserving or orientation-reversing). So the preimage of $\left\{c_{i}\right\}$ in $\widetilde{S}$ is an infinite configuration of topological lines, while the preimage of $\left\{c_{i}\right\}$ in $S^{\alpha}$ is a configuration of lines and curves (the liftings of the $c_{i}$ 's representing powers of $\alpha$, if any). The curves in $S^{\alpha}$ will be denoted by $c_{i}^{\alpha}$ and the lines in $S^{\alpha}$ or $\tilde{S}$ by $\widetilde{c}_{i}$.

According to [1] and [2], $\left\{c_{i}\right\}$ is a minimal configuration in $S$ if and only if for each $S^{\alpha}$ the curves $c_{i}^{\alpha}$ intersect minimally and intersect the lines $\widetilde{c}_{i}$ minimally, that is:

(a) If $\alpha$ is orientation-preserving then the curves representing $\alpha$ in $S^{\alpha}$ are embedded and disjoint, and intersect each line in at most 1 point.

(b) If $\alpha$ is orientation-reversing then the curves representing $\alpha^{r}, r$ odd, have $r-1$ self-intersections and intersect the curves representing $\alpha^{s}$ (s odd, $s \geqslant r$ ) in $r$ points. The curves representing $\alpha^{2}$ are embedded and disjoint from all the other curves representing powers of $\alpha$. A curve representing $\alpha^{r}$ ( $r=2$ or odd) intersects a line that crosses $S^{\alpha}$ in $r$ points.

Case 1 All $c_{i}$ 's are primitive and orientation-preserving.

A natural candidate for a good measuring collection consists of a pair of 'parallel' curves $\mu_{i+}$ and $\mu_{i-}$ for each $c_{i}$, one to the right and one to the left of $c_{i}$ and sufficiently close so that the immersed annulus determined by $\mu_{i+}$ and $\mu_{i-}$ intersects the curves of the configuration along arcs that cross the annulus, and the only multiple points of the configuration inside the annulus are the ones along $c_{i}$. So $c_{i}$ and $\mu_{i+}$ intersect each $c_{j}$ the same number of times (the arcs of intersection between the curves and the annulus give a one to one correspondence between the intersections along $c_{i}$ and the intersections along $\mu_{i+}$ ) and so $\mu_{i+}$ intersects each $c_{j}$ minimally. By construction $\left\{\mu_{i+}, \mu_{i-}\right\}$ meets all the arcs of the configuration. Notice that taking all the widths equal to $\frac{1}{2}$ makes the length of each $c_{i}$ equal to the number of intersections of the configuration along $c_{i}$ (counted with multiplicity). 
Define the distance between two lines in the configuration $\left\{\widetilde{c}_{i}\right\}$ in $\widetilde{S}$ as the minimum number of complementary regions that one has to cross to go from one line to the other (so the distance is 0 iff the lines meet). We will say that 2 -not necessarily different- curves $c_{i}$ and $c_{j}$ in $S$ are close neighbors if two of their preimages $\widetilde{c}_{i}$ and $\widetilde{c}_{j}$ are at distance 1 in $\widetilde{S}$.

Claim In case $1,\left\{\mu_{j+}, \mu_{j-}\right\}$ is a good measuring collection for $\left\{c_{i}\right\}$ if and only if every $c_{i}$ has close neighbors on both sides.

Proof Observe that a polygonal curve $p$ homotopic to $c_{i}$ intersects $\left\{\mu_{i+}, \mu_{i-}\right\}$ minimally if and only if in the corresponding covering $S^{\alpha}$, the curves $p^{\alpha}$ and $c_{i}^{\alpha}$ intersect the same measuring curves and lines $\left(\mu_{i+}^{\alpha}, \mu_{i-}^{\alpha}, \widetilde{\mu}_{j+}, \widetilde{\mu}_{j-}\right)$ and do so the same number of times (once in this case). So $p^{\alpha}$ cannot cross or touch $c_{i}^{\alpha}$ or any other curve $c_{j}^{\alpha}$ (because $p^{\alpha}$ would intersect $\mu_{i+}^{\alpha}, \mu_{i-}^{\alpha}, \mu_{j+}^{\alpha}$ or $\mu_{j-}^{\alpha}$ ), and the annulus bounded by $c_{i}^{\alpha}$ and $p^{\alpha}$ must intersect the lines $\widetilde{c}_{j}$ along arcs that cross the annulus (if a line $\widetilde{c}_{j}$ touches this annulus at one point or intersects it along an arc that starts and ends in $p^{\alpha}$, then $p^{\alpha}$ intersects one of the lines $\widetilde{\mu}_{j+}$ or $\widetilde{\mu}_{j-}$ twice). In particular $p^{\alpha}$ must be made exclusively of arcs of lines $\widetilde{c}_{j}$ that $\operatorname{cross} c_{i}^{\alpha}$.

If all the $c_{i}$ 's representing $\alpha$ have close neighbors on both sides, then the curves $c_{i}^{\alpha}$ in $S^{\alpha}$ are just one complementary region away from other $c_{j}^{\alpha}$ 's or from lines $\widetilde{c}_{j}$ that don't meet $c_{i}^{\alpha}$. So any polygonal curve $p^{\alpha}$ must cross or at least touch one of these curves or lines, and so $p$ cannot have minimal intersections with $\left\{\mu_{j+}, \mu_{j-}\right\}$.

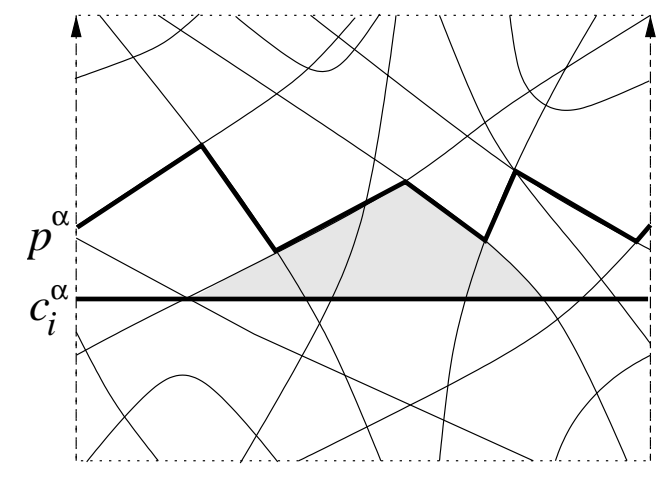

Figure 3

Now suppose that some $c_{i}$ representing $\alpha$ doesn't have close neighbors on one side. Then for each complementary region $R$ on one side of $c_{i}^{\alpha}$, the lines 
adjacent to $R$ must intersect $c_{i}^{\alpha}$. These lines determine triangles with base in $c_{i}^{\alpha}$ that contain $R$, and the widest of these triangles (the one with maximal base in $c_{i}^{\alpha}$ ) is crossed by the lines $\widetilde{c}_{j}$ along arcs that meet the base of the triangle. See figure 3. The union of these wide triangles on one side of $c_{i}^{\alpha}$ is an annulus whose boundaries are $\widetilde{c}_{i}$ and a polygonal curve $p^{\alpha}$, and the lines $\widetilde{c}_{j}$ can intersect this annulus only along arcs that cross the annulus, so $p^{\alpha}$ projects to a polygonal curve $p$ homotopic to $c_{i}$ that intersects $\left\{\mu_{i+}, \mu_{i-}\right\}$ minimally, and $p$ is the nearest polygonal curve with this property.

In the configuration of figure $4 \mathrm{a}$ the curve $c_{1}$ has close neighbors on both sides but the curve $c_{2}$ doesn't. So a metric $g$ that makes each arc of the configuration of length 1 can make $c_{1}$ (but not $c_{2}$ ) a shortest geodesic in the surface.
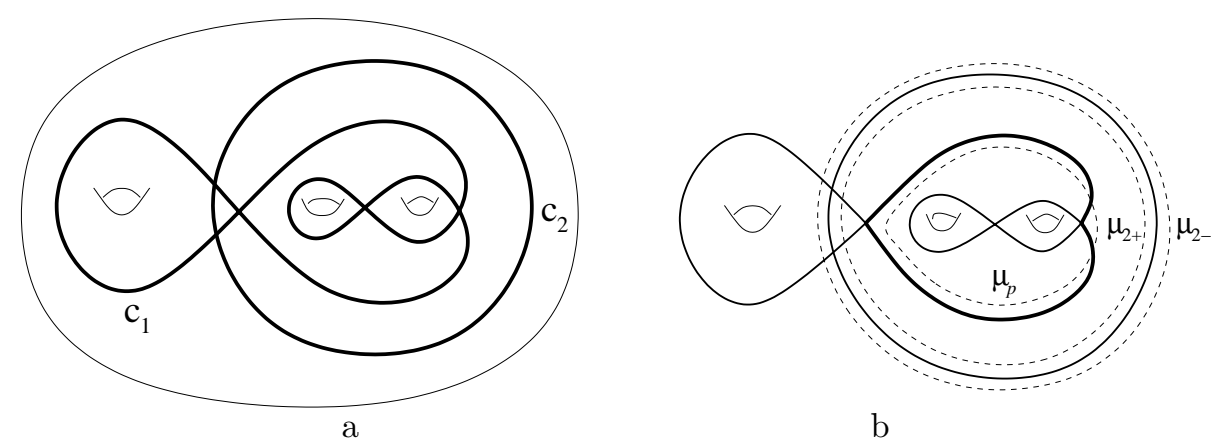

Figure 4

Now we want to extend $\left\{\mu_{i+}, \mu_{i-}\right\}$ to a good measuring collection in the case that some $c_{i}$ 's don't have close neighbors. The idea is given in figure $4 \mathrm{~b}$ : if there is a polygonal curve $p$ homotopic to $c_{i}$ that intersects $\left\{\mu_{i+}, \mu_{i-}\right\}$ minimally, take a measuring curve $\mu_{p}$ that runs "quasiparallel" to $p$ crossing each edge of $p$ once, so its lifting to $S^{\alpha}$ looks like in figure 5 , making it sufficiently close so that each $c_{j}$ intersects the singular annulus determined by $c_{i}$ and $\mu_{p}$ along arcs that cross the annulus.

To see that $\mu_{p}$ intersects each $c_{j}$ minimally, it is enough to show that $\mu_{p}$ and $c_{i}$ intersect each $c_{j}$ the same number of times. This happens because in $S^{\alpha}$ each line $\widetilde{c}_{j}$ intersects the annulus determined by $c_{i}^{\alpha}$ and $\mu_{p}^{\alpha}$ along arcs that cross the annulus (an arc of intersection of $\widetilde{c}_{j}$ with the annulus cannot start and end in $\mu_{p}^{\alpha}$, because then $\widetilde{c}_{j}$ would cross $p^{\alpha}$ twice or it would touch it at one point). So by adding a measuring curve $\mu_{p}$ for each short polygonal curve $p$ we can extend $\left\{\mu_{i+}, \mu_{i-}\right\}$ to a good measuring collection. As only finitely many polygonal curves homotopic to $c_{i}$ can intersect $\left\{\mu_{i+}, \mu_{i-}\right\}$ minimally (because 


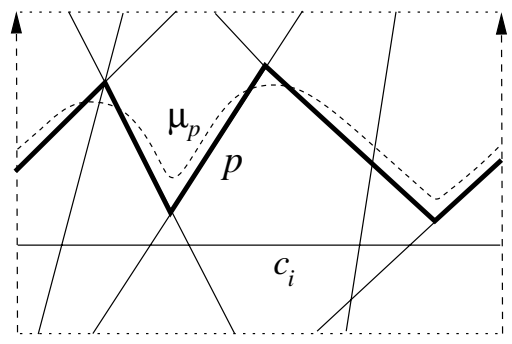

Figure 5

the number of arcs in such polygonals is bounded above by the number of intersections along $c_{i}$ ), we are done.

Case 2 All orientation-reversing $c_{i}$ 's are primitive and no two of them are homotopic

Choose the measuring curves corresponding to the orientation-preserving curves as in case 1 . The orientation-reversing $c_{i}$ 's are one sided, so instead of two parallel curves $\mu_{i+}$ and $\mu_{i-}$ there is a single curve $\mu_{i \pm}$ homotopic to $c_{i}^{2}$ that runs on "both sides" of $c_{i}$. To see that $\mu_{i \pm}$ intersects each $c_{j}$ minimally, look at the covering $S^{\alpha}$ of $S$ corresponding to the class $\alpha$ represented by $c_{i}$. The only closed curve in $S^{\alpha}$ is $c_{i}^{\alpha}$, which by construction does not meet $\mu_{i \pm}^{\alpha}$, and the lines $\widetilde{c}_{j}$ that cross $S^{\alpha}$ intersect $c_{i}^{\alpha}$ at a single point, so they must intersect $\mu_{i \pm}^{\alpha}$ at exactly two points. Now if $p$ is any polygonal curve homotopic to $c_{i}$, then (as $c_{i}$ is one sided) $p^{\alpha}$ must cross $c_{i}^{\alpha}$, so $p^{\alpha}$ must intersect $\mu_{i \pm}^{\alpha}$, and so $\mu_{i \pm}$ doesn't intersect $p$ minimally. Therefore this measuring collection is already good.

Observe that if an orientation-reversing $c_{i}$ is nonprimitive, or is homotopic to another $c_{j}$, then $\mu_{i \pm}$ doesn't have minimal intersection with $c_{i}$ (or $c_{j}$ ), and therefore $\mu_{i \pm}$ cannot be used as a measuring curve.

Case $3 S$ is a projective plane

All $c_{i}$ 's are homotopic to the unique nontrivial element of $\pi_{1}(S)$, so they are embedded and intersect each other in 1 point. For each $c_{i}$ take a collection of measuring curves $\mu_{i x}$ each made of an arc that runs parallel to $c_{i}$ all the way around and a small arc that crosses $c_{i}$ at one point, as in figure $6 \mathrm{a}$.

Make $\mu_{i x}$ sufficiently close to $c_{i}$ so that the other $c_{i}$ 's intersect the singular strip determined by $c_{i}$ and $\mu_{i x}$ along arcs that cross it from $c_{i}$ to $\mu_{i x}$ (so $\mu_{i x}$ will intersect each $c_{j}$ once) and the only multiple points of the configuration 
inside the band are the ones along $c_{i}$. Take two $\mu_{i x}$ for each arc of $c_{i}$, one crossing the arc in each direction. Now if $p$ is any polygonal curve homotopic to $c_{i}$ and $p$ contains arcs of $c_{j}$, then some $\mu_{j x}$ crosses a corner of $p$ twice (see figure $6 \mathrm{~b}$ ), so $p$ doesn't have minimal intersection with that $\mu_{j x}$.
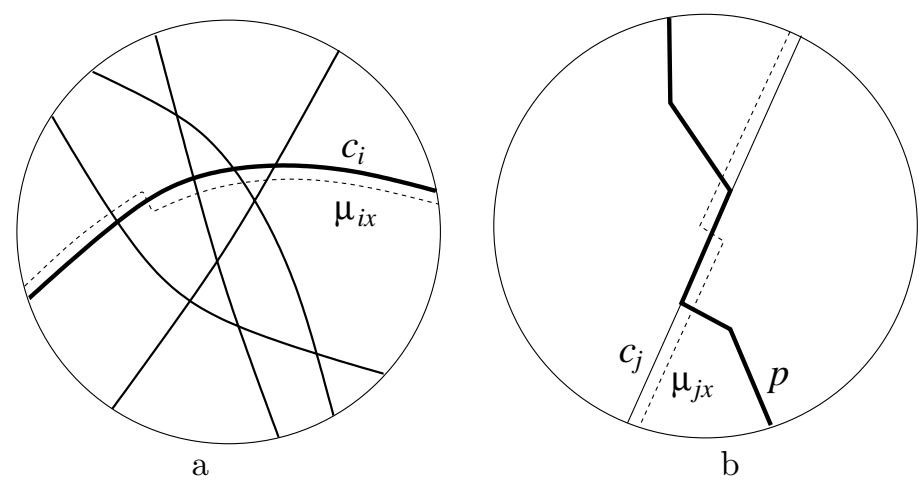

Figure 6

Case 4 All orientation-reversing $c_{i}$ 's are primitive, but some are homotopic

Choose the measuring curves for the orientation-preserving $c_{i}$ 's as in case 1 , and those for the orientation-reversing $c_{i}$ 's that are not homotopic to other $c_{j}$ 's as in case 2 .

Now consider an orientation-reversing class $\alpha$ represented by 2 or more $c_{i}$ 's. These $c_{i}$ 's lift to curves $c_{i}^{\alpha}$ in the Moebius band $S^{\alpha}$ that are embedded and intersect each other in 1 point. For each of these $c_{i}$ 's take a collection of measuring curves $\mu_{i x}$ as in case 3 , each one made of an arc that runs parallel to $c_{i}$ all the way around and a small arc that crosses $c_{i}$ at one point, so $\mu_{i x}$ lifts to a curve $\mu_{i x}^{\alpha}$ in $S^{\alpha}$ that intersects $c_{i}^{\alpha}$ in exactly one point as in figure 7a. Take again one $\mu_{i x}$ crossing each arc of $c_{i}$ in each direction. As $\mu_{i x}$ and $c_{i}$ intersect each $c_{j} \neq c_{i}$ the same number of times, then $\mu_{i x}$ intersects each $c_{j} \neq c_{i}$ minimally, and as $\mu_{i x}$ intersects $c_{i}$ one more time than $c_{i}$ intersects itself, then $\mu_{i x}$ also intersects $c_{i}$ minimally.

Observe that a polygonal curve $p$ that intersects these $\mu_{i x}$ 's minimally must be made exclusively of arcs of orientation-preserving $c_{j}$ 's, because if $p$ contains an arc of some orientation-reversing $c_{j}$ then one of the $\mu_{j x}$ 's crosses a corner of $p$ twice. And one can show as in case 1 that such $p$ has minimal intersection with the curves $\mu_{i+}, \mu_{i-}$ and $\mu_{i x}$ if and only if $p^{\alpha}$ intersects $c_{i}^{\alpha}$ and every curve $c_{j}^{\alpha}$ homotopic to $c_{i}^{\alpha}$ in exactly one point, and each line $\widetilde{c}_{j}$ that intersects the singular annulus determined by $c_{i}^{\alpha}$ and $p^{\alpha}$ does so along one arc that crosses the annulus from $p^{\alpha}$ to $c_{i}^{\alpha}$. 


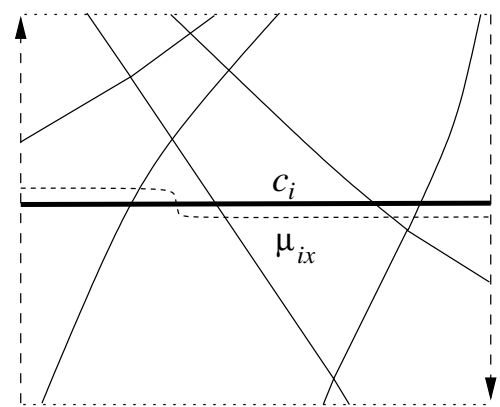

a

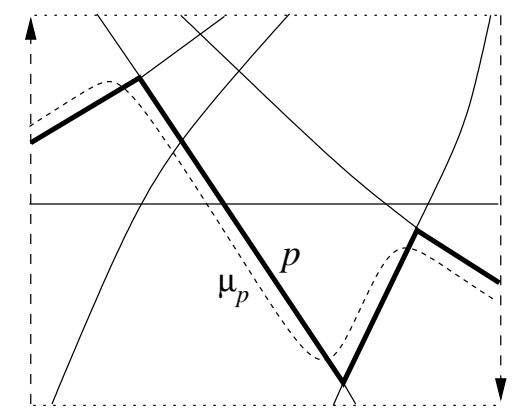

$\mathrm{b}$

Figure 7

If there is a short polygonal curve $p$ homotopic to $\alpha$, take a measuring curve $\mu_{p}$ that runs "quasiparallel" to $p$ crossing each edge of $p$ once, so $\mu_{p}$ lifts to a curve $\mu_{p}^{\alpha}$ in $S^{\alpha}$ that looks like in figure $7 \mathrm{~b}$. To see that $\mu_{p}$ intersects each $c_{j}$ minimally, observe that $\mu_{p}^{\alpha}$ intersects each curve $c_{j}^{\alpha}$ once (otherwise $p^{\alpha}$ would intersect $c_{j}^{\alpha}$ more than once) and that each $\widetilde{c}_{j}$ intersects the singular annulus determined by $c_{i}^{\alpha}$ and $\mu_{p}^{\alpha}$ along arcs that cross it from $c_{i}^{\alpha}$ to $\mu_{p}^{\alpha}$ (an arc of intersection cannot start and end in $\mu_{p}$, because then $\widetilde{c}_{j}$ would intersect the singular annulus determined by $c_{i}^{\alpha}$ and $p^{\alpha}$ in an arc that starts and ends in $\left.p^{\alpha}\right)$.

By construction the number of intersections between $\mu_{p}^{\alpha}$ and $p^{\alpha}$ is equal to the number of corners of $p$, so $\mu_{p}$ intersects $p$ minimally only when $p$ has one corner. To deal with these short polygonal curves with only one corner, we need an extra measuring curve $\mu_{\alpha \pm}$ whose lifting to $S^{\alpha}$ runs parallel to the boundary of the region $V \alpha$ determined by all the curves $c_{i}^{\alpha}$, as in figure $8 \mathrm{a}$, so $\mu_{\alpha \pm}$ is homotopic to $\alpha^{2} . \mu_{\alpha \pm}$ intersects each $c_{j}$ minimally because the curves $c_{i}^{\alpha}$ are contained in the Moebius band bounded by $\mu_{\alpha \pm}$, and if a line $\widetilde{c}_{j}$ intersects this Moebius band along a nonessential arc then $\widetilde{c}_{j}$ intersects some $c_{i}^{\alpha}$ in two points.

We claim that if $p$ is a short polygonal curve with one corner then $p^{\alpha}$ cannot be contained in $V \alpha$, so $p^{\alpha}$ intersects $\mu_{\alpha \pm}$ and so $p$ doesn't intersect $\mu_{\alpha \pm}$ minimally. If $p^{\alpha}$ were contained in $V \alpha$ then its corner would be in the region determined by two curves $c_{i}^{\alpha}$ and $c_{j}^{\alpha}$. As $p^{\alpha}$ is made of an arc of a line $\widetilde{c}_{k}$ that starts and ends at the corner, $\widetilde{c}_{k}$ would have to cross $c_{i}^{\alpha}$ or $c_{j}^{\alpha}$ twice (see figure $8 \mathrm{~b})$ contradicting the fact that $c_{k}$ intersects $c_{i}$ and $c_{j}$ minimally.

Case 5 Some orientation-reversing $c_{i}$ 's are nonprimitive

Let $1 \leq r_{1}<r_{2}<\ldots<r_{n}$ be the odd powers of a primitive orientationreversing class $\alpha$ represented by $c_{i}$ 's in the configuration. Each of these $c_{i}$ 's 

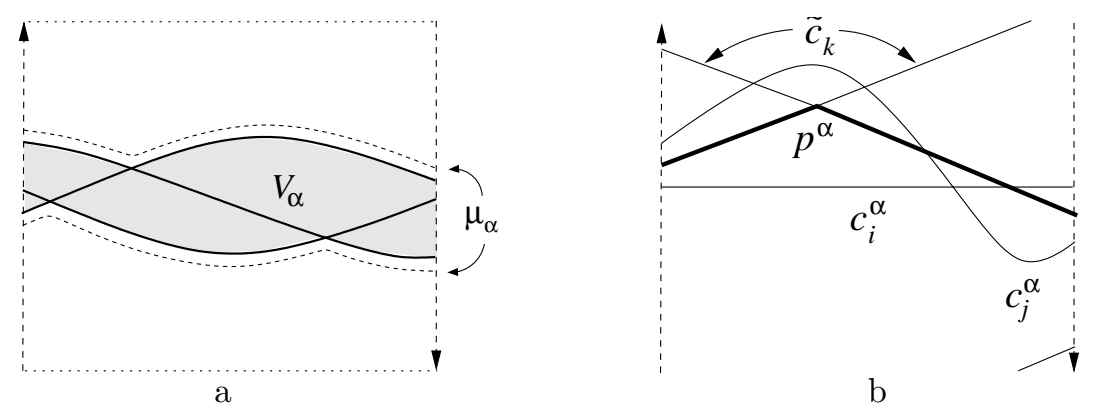

Figure 8

lifts to an immersed curve $c_{i}^{\alpha}$ in the Moebius band $S^{\alpha}$. For each of these $c_{i}$ 's take a collection of measuring curves $\mu_{i x}$ as in case 4 , each made of an arc that runs parallel to $c_{i}$ all the way around and a small arc that crosses $c_{i}$ at one point. So each $\mu_{i x}$ intersects every $c_{j}$ minimally and every polygonal curve $p$ that intersects these $\mu_{i x}$ 's minimally is made of arcs of orientation-preserving $c_{j}$ 's For each polygonal curve $p$ homotopic to $c_{i}$ that intersects these $\mu_{i x}$ 's minimally, take a measuring curve $\mu_{p}$ that runs quasiparallel to $p$ crossing each edge of $p$ once so, as in case $4, \mu_{p}$ intersects every $c_{j}$ minimally, but $\mu_{p}$ intersects $p$ minimally only when $p$ has one corner.

To deal with these short polygonal curves with one corner representing $\alpha^{r_{k}}$, we need to add an extra measuring curve $\mu_{\alpha \pm}$ homotopic to $\alpha^{2}$ and measuring curves $\mu_{\alpha k}$ homotopic to $\alpha^{r_{k+1}}$ for each $k<n$. One can show as in case 4 that the polygonal curves with one corner representing $\alpha^{r_{k}}$ cannot be contained in the region $V \alpha^{r_{k}}$ of $S^{\alpha}$ determined by the images of all the $c_{i}^{\alpha}$ 's representing $\alpha^{r_{k}}$. The minimal intersection of the curves in $S^{\alpha}$ implies that all the curves representing $\alpha^{r_{k}}$ are contained in the region determined by each curve representing $\alpha^{r_{k+1}}$, so $V \alpha^{r_{k}} \subset V \alpha^{r_{k+1}}$, each curve representing $\alpha^{r_{k+1}}$ intersects $V \alpha^{r_{k}}$ along one arc, and each line that intersects $V \alpha^{r_{k}}$ does so along one essential arc.

Let $\mu_{\alpha \pm}$ be a curve whose lifting to $S^{\alpha}$ runs parallel to the boundary of $V \alpha^{r_{n}}$, so $\mu_{\alpha \pm}$ is homotopic to $\alpha^{2}$. Then $\mu_{\alpha \pm}$ intersects every $c_{j}$ minimally, but any polygonal curve representing an odd power of $\alpha$ that intersects $\mu_{\alpha \pm}$ minimally must be contained in $V \alpha^{r_{n}}$. Now for each $k<n$, choose a curve $c_{i}^{\alpha}$ representing $\alpha^{r_{k+1}}$ which is closest to $V \alpha^{r_{k}}$ in the sense that the region determined by its image does not contain any other $c_{j}^{\alpha}$ representing $\alpha^{r_{k+1}}$. Let $\mu_{\alpha k}$ be a curve whose lifting to $S^{\alpha}$ runs parallel to the arc $c_{i}^{\alpha} \cap V \alpha_{r_{k}}$ and then runs around the boundary of $V \alpha_{r_{k}}$ enough times to complete a curve homotopic to $\alpha^{r_{k+1}}$. 
Figure 9a shows a lifting of $\mu_{\alpha k}$ to $S^{\alpha^{r} k+1}$. To prove that $\mu_{\alpha k}$ intersects each $c_{j}$ minimally, it is enough to show that in the covering $S^{\alpha^{r} k+1}$ the preimages of $c_{j}$ intersect the region determined by the liftings of $c_{i}$ and $\mu_{\alpha k}$ along arcs that cross that region. An arc of intersection $a$ that didn't cross that region would look as in figure $9 \mathrm{~b}$, but this arc cannot belong to a line $\widetilde{c}_{j}$ because then $\widetilde{c}_{j}$ would intersect $V \alpha^{r_{k}}$ in at least two arcs, and it cannot belong to a curve $c_{j}^{\alpha}$ representing $\alpha^{r_{k}}$ or a smaller power of $\alpha$ because these curves are contained in $V \alpha^{r_{k}}$. So the arc $a$ must belong to a curve $c_{j}^{\alpha}$ representing some larger power of $\alpha$, and so $c_{j}^{\alpha}=a \cup a^{\prime}$, where $a^{\prime}$ is an arc in $V \alpha^{r_{k}}$. So $c_{j}^{\alpha}$ lies in the region determined by $c_{i}^{\alpha}$, but by the choice of $c_{i}^{\alpha}$ no curve representing $V \alpha^{r_{k+1}}$ or a larger power of $\alpha$ can be contained in this region.

Now if $p$ is a polygonal curve with one corner representing $\alpha^{r_{k}}$ then its lifting to $S^{\alpha}$ is not contained in $V \alpha^{r_{k}}$, so it is not contained in the region determined by $\mu_{\alpha k}$, so $p$ does not intersect $\mu_{\alpha k}$ minimally.

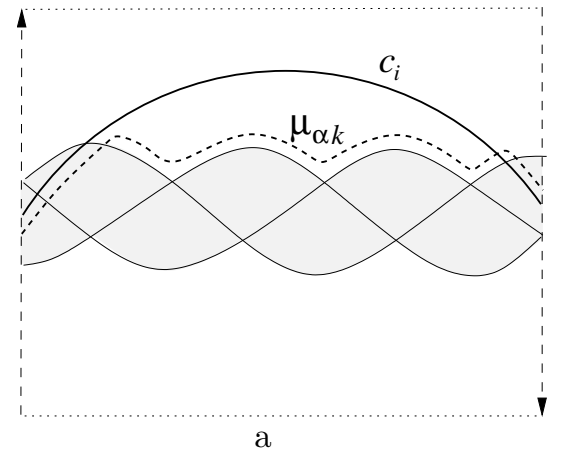

$\mathrm{a}$

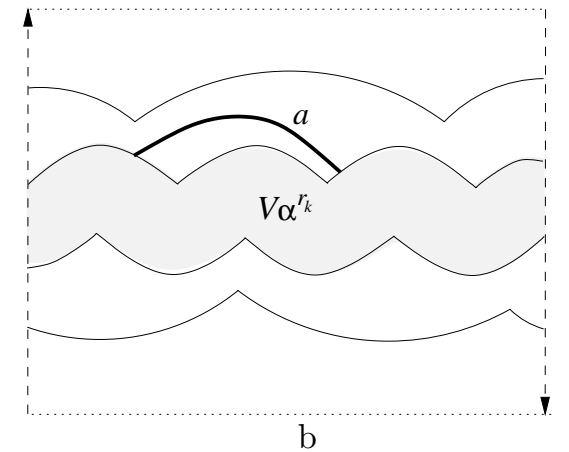

$\mathrm{b}$

Figure 9

\section{Choice of widths}

The measuring collection for $\left\{c_{i}\right\}$ constructed above is made of curves homotopic to some $c_{i}\left(\mu_{i+}, \mu_{i-}, \mu_{i \times}, \mu_{p}\right.$ and $\left.\mu_{\alpha k}\right)$ or the square of some primitive orientation-reversing class $\alpha\left(\mu_{i \pm}\right.$ and $\left.\mu_{\alpha \pm}\right)$. The choice of widths to prove the second part of the theorem is not obvious because the minimum number of self-intersections of an orientation-reversing $c_{i}$ differs from the minimum number of intersections between $c_{i}$ and a homotopic curve by 1 . The condition that the length of each $c_{i}$ in the configuration must be equal to the number of intersections along it gives a system of linear equations on the widths of the measuring curves that has a unique solution for the sums of widths of the measuring curves in each homotopy class: 
(a) Make the sum of the widths of the measuring curves $\left(\mu_{i+}, \mu_{i-}\right.$ and $\mu_{p}$ 's) in each orientation-preserving class equal to the number of $c_{i}$ 's in that class.

(b) If $\alpha$ is a primitive orientation-reversing class, and $1 \leq r_{1}<r_{2}<\ldots$ are the odd powers of $\alpha$ represented by some $c_{i}$ 's, make the sum of the widths of the measuring curves ( $\mu_{i x}, \mu_{p}$ and $\mu_{\alpha k-1}$ 's) representing $\alpha^{r_{k}}, k>1$, equal to the number of $c_{i}$ 's representing that class, but for the measuring curves representing $\alpha^{r_{1}}$ make the sum of their widths $\frac{1}{r_{1}}$ units less than the number of $c_{i}$ 's representing that class. Finally, make the width of each measuring curve $\left(\mu_{i \pm}\right.$ and $\left.\mu_{\alpha \pm}\right)$ representing $\alpha^{2}$ equal to $\frac{1}{2}$.

A problem arises when $r_{1}=1$ and only 1 curve $c_{i}$ represents $\alpha^{r_{1}}$, because then the sum of the widths of the measuring curves homotopic to $\alpha$ is 0 , which means that these measuring curves cannot be used, and the rest of the measuring collection may not be good. This can be arranged by replacing the measuring curves representing $\alpha$ by suitable curves representing $\alpha^{r_{2}}$ as follows:

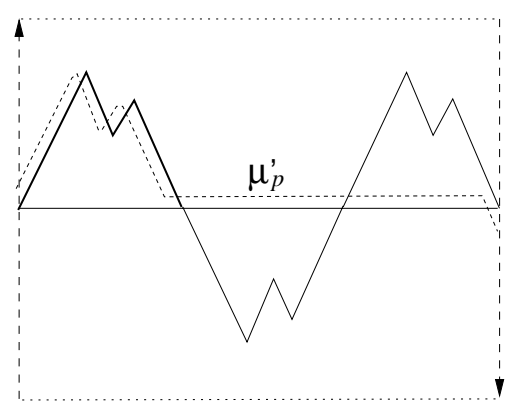

Figure 10

Trade each curve $\mu_{i x}$ made of an arc that goes once around $c_{i}$ and a small arc that crosses $c_{i}$ at one point, for a curve $\mu_{i x}^{\prime}$ made of an arc that goes $r_{2}$ times around $c_{i}$ and the small arc. And trade each $\mu_{p}$ representing $\alpha$ for a curve $\mu_{p}^{\prime}$ obtained by replacing the small arc of $\mu_{p}$ that crosses $c_{i}$ by an arc that goes $r_{2}-1$ times around $c_{i}$ so it now represents $\alpha^{r_{2}}$ (figure 10 shows the lifting of $\mu_{p}^{\prime}$ to $\left.S^{\alpha^{r_{2}}}\right)$. It is not hard to see that $\mu_{i x}^{\prime}$ and $\mu_{p}^{\prime}$ intersect each $c_{j}$ minimally, but intersect nonminimally all the polygonal curves that intersect $\mu_{i x}$ or $\mu_{p}$ nonminimally. This proves the second part of the theorem.

Figure 11 shows the lengths of the arcs in the configuration in figure 4 resulting from making $\mu_{i+}$ and $\mu_{1-}$ of width $\frac{1}{2}$ and $\mu_{2-}, \mu_{2+}$ and $\mu_{p}$ of width $\frac{1}{3}$.

Remark Theorem 1.1 clearly holds for nonclosed surfaces, provided that the curves don't meet the boundary. It also works for minimal configurations of 


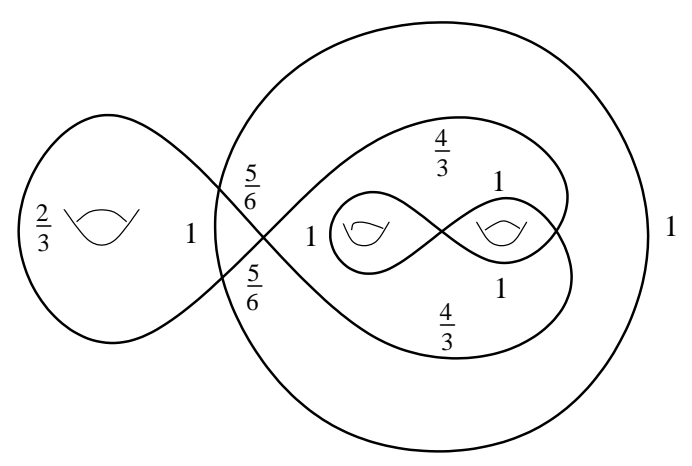

Figure 11

properly immersed curves and arcs in a surface with boundary, considering either minimal configurations with the endpoints of the arcs fixed or free to move along $\partial S$ (one just needs to use measuring arcs analogous to the measuring curves).

Theorem 1.1 contrasts with the examples of Hass and Scott [4] of minimal configurations of primitive and nonhomotopic curves in a surface which are not configurations of geodesics for any metric of negative curvature on the surface. These configurations, however, can be realized by metrics of nonpositive curvature.

Questions Which configurations of primitive curves in a surface are configurations of shortest geodesics for metrics of negative curvature? and for metrics of non positive curvature?

The second part of theorem 1.1 is only significant for configurations containing more than 1 curve. For configurations of 1 curve in general position one may ask if the lengths of all the arcs can be made equal (we know that the answer is yes if the curve is orientation-reversing, and no in general if the configuration is not in general position or contains more than one curve). One may also ask if every minimal configuration of curves in general position is contained in a configuration of shortest geodesics in which each arc has the same length. These questions are equivalent to the following:

Questions Do all minimal 1-curve configurations have close neighbors? Can every minimal configuration be extended to a configuration with close neighbors?

One may face strong restrictions when trying to extend a minimal configuration to contain other curves. For example, if one wants to extend the configuration 


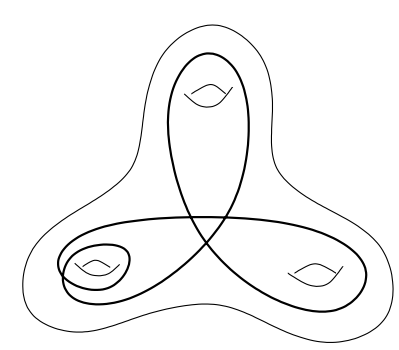

Figure 12

in figure 12 to one containing a curve in the homotopy class of figure 1 , then the first curve must look as in figure 1b. Theorem 1.1 implies that some extension is always possible:

Corolary 1.5 Every minimal configuration of curves in $S$ can be extended to a minimal configuration containing curves in any given homotopy classes in $S$.

Denote by $l_{g}(a)$ the minimum length in the free homotopy class of the curve $a$ when $S$ is given a Riemannian metric $g$. Denote by $a \bigcap b$ the minimum number of intersections between curves in the free homotopy classes of $a$ and $b$, and by $a \bigcap a$ the minimal number of self-intersections in the homotopy class of $a$.

Corolary 1.6 If $l_{g}(a) \leq k \cdot l_{g}(b)$ for every Riemannian metric $g$ on $S$, then $a \bigcap c \leq k \cdot b \bigcap c$ for every curve $c$ in $S$. In particular, $a \bigcap a \leq k \cdot a \bigcap b \leq k^{2} \cdot b \bigcap b$.

Proof Suppose that $a \bigcap c>k \cdot b \bigcap c$ for some curve $c$. We may assume that $a, b$ and $c$ are in general position and have minimal intersection and selfintersection. Apply the proof of theorem 1.1 to the configuration formed by $a$ and $b$, but add to the resulting measuring collection a copy of the curve $c$ with weight $w$. If

$$
w>\frac{k \cdot b \bigcap b+(k-1) \cdot a \bigcap b-a \bigcap a}{a \bigcap c-k \cdot b \bigcap c}
$$

then for the resulting metric $g$ we have

$$
l_{g}(a)=a \bigcap a+a \bigcap b+w \cdot a \bigcap c>k \cdot(b \bigcap a+b \bigcap b+w \cdot b \bigcap c)=k \cdot l_{g}(b)
$$

contrary to the hypothesis that $l_{g}(a) \leq k \cdot l_{g}(b)$.

Corollary 1.6 clearly holds if the curves $a, b$ and $c$ are replaced by any finite families of curves or arcs. 


\section{Cutting and pasting.}

Let $\left\{a_{i}\right\}$ be a configuration of curves with transverse intersections in $S$. A cut and paste on $\left\{a_{i}\right\}$ is done by cutting these curves at some of their intersection points and glueing the resulting arcs in a different order to obtain a new collection of curves $\left\{b_{j}\right\}$. These curves have some 'corners' that can be rounded so the total number of intersections and the total length of the original configuration are reduced.

Lemma 2.1 If a collection of curves $\left\{a_{i}\right\}$ in $S$ can be cut and pasted to obtain the collection $\left\{b_{j}\right\}$, and $\left\{a_{i}\right\}$ can be homotoped to a collection $\left\{a_{i}^{\prime}\right\}$ without removing any intersection points in the process, then $\left\{a_{i}^{\prime}\right\}$ can be cut and pasted to obtain a collection homotopic to (the nontrivial) $\left\{b_{j}\right\}$.

Proof We want to show that the instructions for cutting and pasting $\left\{a_{i}\right\}$ to get $\left\{b_{j}\right\}$ can be transmitted along the homotopy from $\left\{a_{i}\right\}$ to $\left\{a_{i}^{\prime}\right\}$ so that the final result is homotopic to $\left\{b_{j}\right\}$. This is not obvious even though the intersection points of $\left\{a_{i}\right\}$ can be traced along the homotopy (they don't disappear), because the result of doing the "same" cut and paste before or after the homotopy may be different, as shown in figure 13 .

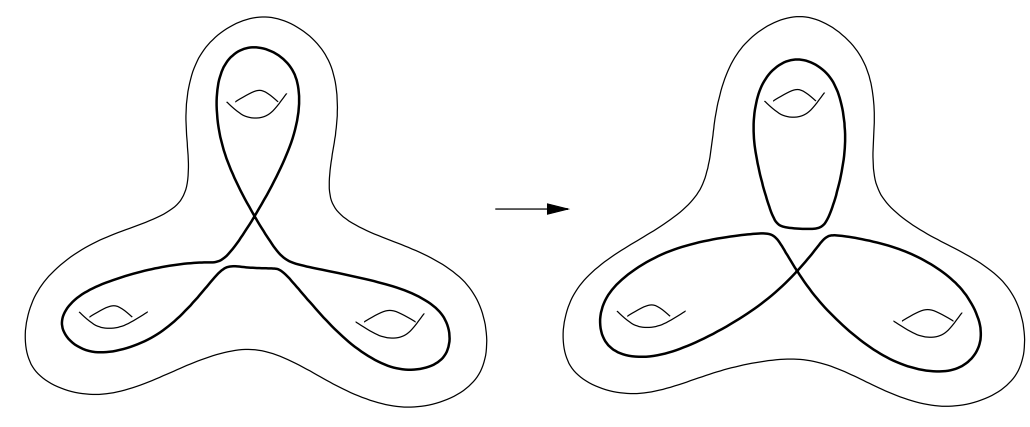

Figure 13

Any homotopy that doesn't remove intersection points can be done using 3 types of local moves in the configuration. The first two moves, adding a small loop and creating a small bigon, do not change the homotopy class of the resulting curves. Nevertheless, when doing these moves one can add cut and paste instructions at the new intersections to avoid increasing the number of intersections of the resulting curves (see figure 14a,b).

In the third move a local configuration of $n$ arcs that intersect each other at different points collapses into one where all the arcs meet at a single point, 


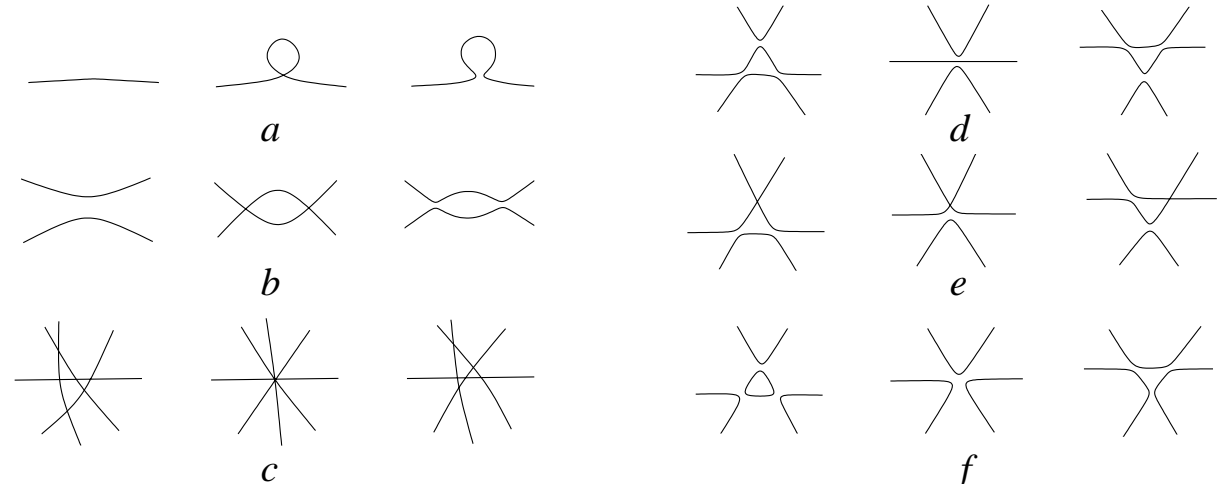

Figure 14

or viceversa: a configuration with a multiple intersection opens up (see figure 14c). Observe that to transmit some cut and paste instructions during these local moves one only needs that the endpoints of the arcs that were connected by the original cut and paste instructions get connected by the new instructions (any curve contained in the local configuration is trivial). As the endpoints of the arcs that meet at a single point can be connected at will by cutting and pasting at that point, then all cut and paste instructions can be transmitted when a local configuration collapses into a multiple intersection.

So the problem is to transmit the cut and paste instructions when a multiple intersection opens up. In the case $n=3$ one can see how this can be done directly (figure 14d-f shows some cases). Observe that the new instructions may not be unique, but they can always be chosen to avoid creating new curves and to avoid increasing the number of intersections of the resulting curves. In the case $n>3$, modify the homotopy so the multiple intersection opens up one arc at a time. If an arc a moves away from the multiple intersection point and the cut and paste instructions don't change, then the only connections that are affected are those involving the endpoints of $a$, which are connected to the endpoints of at most 2 other arcs of the local configuration. But we can change the cut and paste instructions at the intersections of these 3 arcs as in the case $n=3$ to get the right connections for the endpoints of $a$, and then change the cutting and pasting instructions at the multiple intersection point as needed to get the right connections between all the other endpoints. Now repeat the argument until the multiple intersection opens up completely.

In [3] Hass and Scott defined a 'curve flow' that takes any configuration of primitive curves in a surface to a configuration of shortest geodesics by a homo- 
topy that does not increase the number of intersections at any moment. This result and the previous lemma imply the following version of the theorem of Freedman, Hass and Scott:

Proposition 2.2 Any finite family of primitive, orientation-preserving curves in $S$ can be cut and pasted to obtain a freely homotopic family of curves with minimal intersections and self-intersections.

Proof By [3] there is a homotopy that takes the family $\left\{a_{i}\right\}$ to some minimal intersection family $\left\{a_{i}^{\prime}\right\}$ without increasing the number of intersections, so running the homotopy backwards we get a homotopy that takes $\left\{a_{i}^{\prime}\right\}$ to $\left\{a_{i}\right\}$ without removing any intersection points. Now lemma 2.1 shows how to transmit the "don't cut anything" instructions in $\left\{a_{i}^{\prime}\right\}$ to cutting and pasting instructions in $\left\{a_{i}\right\}$ without increasing the number of intersections of the resulting curves.

Figure 15 shows a nonminimal configuration of 2 curves and a cut and paste that transforms it into a minimal configuration.
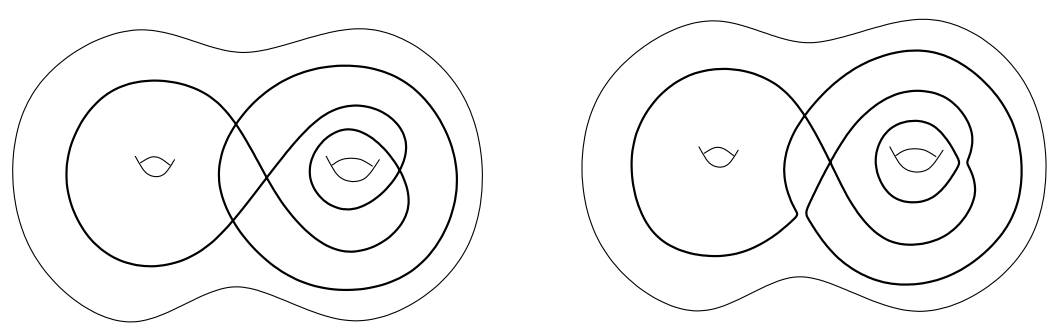

Figure 15

Corolary 2.3 If a collection $\left\{a_{i}\right\}$ of curves with minimal intersection and self-intersection in $S$ can be cut and pasted to obtain the collection $\left\{b_{j}\right\}$, then $l_{g}\left(\left\{a_{i}\right\}\right)>l_{g}\left(\left\{b_{j}\right\}\right)$ for every Riemannian metric $g$ on $S$.

Proof Observe that the hypothesis that $\left\{a_{i}\right\}$ has minimal intersections is essential. If $g$ is a Riemannian metric on $S$ and $\left\{a_{i}^{\prime}\right\}$ is a collection of shortest geodesics (for the metric $g$ ) homotopic to $\left\{a_{i}\right\}$, then by [3] there is a homotopy from $\left\{a_{i}\right\}$ to $\left\{a_{i}^{\prime}\right\}$ that does not increase the number of intersections, so as $\left\{a_{i}\right\}$ already had minimal intersections the number of intersections must remain constant. So by lemma 2.1 the cutting and pasting instructions to get $\left\{b_{j}\right\}$ from $\left\{a_{i}\right\}$ can be transmitted to get a homotopic collection $\left\{b_{j}^{\prime}\right\}$ from $\left\{a_{i}^{\prime}\right\}$, so $l_{g}\left(\left\{a_{i}\right\}\right)=l_{g}\left(\left\{a_{i}^{\prime}\right\}\right)>l_{g}\left(\left\{b_{j}^{\prime}\right\}\right)=l_{g}\left(\left\{b_{j}\right\}\right)$. 
Example The converse to corollary 2.3 is not true. Figure 16 shows 2 curves $a$ and $b$ on a surface such that $a$ cannot be cut and pasted to obtain a curve homotopic to $b$ but one can show that $l_{g}(a)>l_{g}(b)$ for every Riemannian metric $g$ on $S$ (so $a \bigcap c \geq b \bigcap c$ for every curve $c$ ).
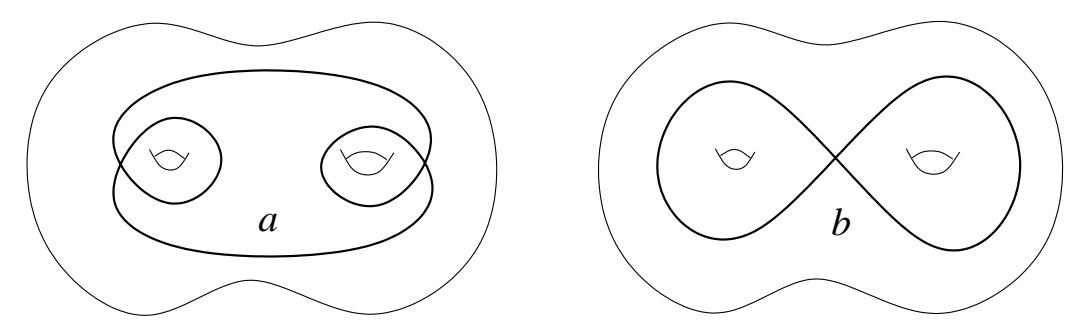

Figure 16

Question If $a \bigcap c \leq b \bigcap c$ for every curve $c$ in $S$, is it true that $l_{g}(a) \leq l_{g}(b)$ for every Riemannian metric $g$ on $S$ ?

\section{References}

[1] M Freedman, J Hass, P Scott, Closed geodesics on surfaces, Bull. London Math. Soc. 14 (1982) 385-391

[2] J Hass, H Rubinstein, One-sided geodesics on surfaces, Michigan Math. Journal 33 (1986) 155-168

[3] J Hass, P Scott, Shortening curves on surfaces, Topology, 33-1 (1994) 25-43

[4] J Hass, P Scott, Configurations of curves and geodesics on surfaces, Geometry and Topology Monographs 2: Proceedings of the Kirbyfest (1999) 201-213

[5] M Shepard, Ph.D. Thesis, U.C. Berkeley (1990)

Instituto de Matemáticas UNAM

Ciudad Universitaria, México D.F. 04510, México

Email: max@math.unam.mx

Received: 8 January 2001 